\title{
Evaluation of a novel rapid TRC assay for the detection of influenza using nasopharyngeal swabs and gargle samples
}

\author{
Norihito Kaku ${ }^{1,2}$ (1) Kohji Hashiguchi ${ }^{3}$. Norihiko Akamatsu ${ }^{2}$. Fumiko Wakigawa ${ }^{4}$. Junichi Matsuda ${ }^{2}$. \\ Kenzo Komaru ${ }^{4}$ - Takumi Nakao ${ }^{3}$ - Yosuke Harada ${ }^{3}$ - Atsuko Hara ${ }^{3,5}$ • Naoki Uno ${ }^{1,2}$ • Kei Sakamoto ${ }^{1,2}$. \\ Yoshitomo Morinaga ${ }^{1,2} \cdot$ Takeshi Kitazaki $^{3}$. Hiroo Hasegawa ${ }^{1,2} \cdot$ Taiga Miyazaki $^{5}$ - Masaaki Fukuda ${ }^{3}$. \\ Koichi Izumikawa $^{6} \cdot$ Hiroshi Mukae $^{5} \cdot$ Katsunori Yanagihara ${ }^{1,2}$
}

Received: 30 September 2020 / Accepted: 9 February 2021 / Published online: 16 February 2021

(C) The Author(s), under exclusive licence to Springer-Verlag GmbH, DE part of Springer Nature 2021

\begin{abstract}
We evaluated a novel transcription-reverse transcription concerted reaction (TRC) assay that can detect influenza A and B within 15 min using nasopharyngeal swab and gargle samples obtained from patients with influenza-like illness, between January and March 2018 and between January and March 2019. Based on the combined RT-PCR and sequencing results, in the nasal swabs, the sensitivity and specificity of TRC for detecting influenza were calculated as 1.000 and 1.000, respectively. In the gargle samples, the sensitivity and specificity of TRC were 0.946 and 1.000 , respectively. The TRC assay showed comparable performance to RT-PCR in the detection of influenza viruses.
\end{abstract}

Keywords Rapid detection · Influenza · TRC method; RT-PCR

\section{Introduction}

The transcription-reverse transcription concerted reaction (TRC) method is a combination of direct and rapid isothermal RNA amplification and real-time identification using an intercalation-activating fluorescence (INAF)

All authors meet the ICMJE authorship criteria.

Norihito Kaku

kaku-ngs@umin.ac.jp

1 Present address: Department of Laboratory Medicine, Nagasaki University Graduate School of Biomedical Sciences, 1-7-1

Sakamoto, Nagasaki 852-8501, Japan

2 Department of Laboratory Medicine, Nagasaki University Hospital, Nagasaki, Japan

3 Department of Respiratory Medicine, Japanese Red Cross Nagasaki Genbaku Hospital, Nagasaki, Japan

4 Department of Clinical Laboratory, Japanese Red Cross Nagasaki Genbaku Hospital, Nagasaki, Japan

5 Department of Respiratory Medicine, Nagasaki University Graduate School of Biomedical Sciences, Nagasaki, Japan

6 Department of Infectious Diseases, Nagasaki University Graduate School of Biomedical Sciences, Nagasaki, Japan probe [1, 2]. In Europe, Japan, and Vietnam, TRC ready-to use regents have been used for the diagnosis of tuberculosis [3], nontuberculous mycobacterial infections, Chlamydia infection, gonorrhea, and mycoplasma pneumonia. Recently, a novel TRC assay that can detect influenza A and B within 15 min was developed $[4,5]$. In this study, we evaluated the efficacy of the novel rapid TRC assay for detecting influenza viruses in nasopharyngeal swab and gargle samples obtained from patients with influenza-like illness.

\section{Materials and methods}

\section{Ethics}

This study was approved by the ethics committee of Nagasaki University Hospital (approval numbers: 17121822 and 18111919) and was registered at the UMIN Clinical Trials Registry (reference numbers: UMIN000032395 and UMIN000034545). Written informed consent for participation in and publication of this study was obtained from all participants before sample collection. 


\section{Study design}

A prospective observational study was conducted in period 1, between January and March 2018, and period 2, between January and March 2019. Patients who visited or were hospitalized at the Department of Respiratory Medicine, Japanese Red Cross Nagasaki Genbaku Hospital, with influenza-like illness (ILI) [6] were included in this study. Patients were excluded if they were administered anti-influenza agents within 1 month prior to the day on which they were sampled. Nasopharyngeal swabs were collected from patients in both periods using two swabs: one was used for antigen testing using silver amplification immunochromatography (FUJI DRI-CHEM IMMUNO AG Cartridge FluAB, Fujifilm, Kanagawa, Japan) [7] at Nagasaki Genbaku Hospital and the other was stored at $-20^{\circ} \mathrm{C}$ until further analysis. Gargle samples were collected from patients in period 2. For gargle sampling, the patients gargled their throat with $20 \mathrm{~mL}$ distilled water for $10 \mathrm{~s}$. The physicians determined the clinical diagnosis with history taking, physical findings, and results of the influenza antigen test. All information, such as clinical report forms and results of the TRC and RT-PCR, was summarized and analyzed at Nagasaki University Hospital.

\section{Sample analysis}

We performed a rapid TRC assay based on the protocol described in Japan-patent (JP,2017-195871, A) at Nagasaki University Hospital. In summary, the procedure was performed as follows. A nasopharyngeal swab or gargle swab was mixed in 1-mL extraction buffer containing surfactant and incubated at $52{ }^{\circ} \mathrm{C}$ for $1 \mathrm{~min}$. Thirty microliters of the sample was mixed with dry reagent containing enzymes, substrates, primers, and INAF probes. The mixture was incubated at $46{ }^{\circ} \mathrm{C}$, and the fluorescence was monitored. RT-PCR and sequencing were performed as a gold standard at Tosoh Corporation based on the Influenza Diagnosis Manual [8-10]. Total RNA was isolated from $140 \mu \mathrm{L}$ of TRC extraction buffer mixed with a nasopharyngeal swab or $140 \mu \mathrm{L}$ of a gargle specimen using the Qiagen RNeasy kit. RT-PCR was performed using the One Step PrimeScript ${ }^{\mathrm{TM}}$ RT-PCR Kit (TAKARA BIO, Shiga, Japan). TRC assay and RT-PCR were performed independently, and the results of them were combined at Nagasaki University Hospital after all analysis was completed. If the results of the TRC and RT-PCR were different, the samples were analyzed by sequencing.

\section{Statistical analysis}

All statistical analyses were performed with EZR version 1.41 (Saitama Medical Center, Jichi Medical University, Saitama, Japan) [11], which is a graphical user interface for $\mathrm{R}$ (the $\mathrm{R}$ Foundation for Statistical Computing, Vienna, Austria; version 3.6.3). Fisher's exact test was used to compare categorical variables, and the statistical significance level was set at $<0.05$. The sensitivity (Se), specificity ( $\mathrm{Sp}$ ), positive predictive value (PPV), and negative predictive value (NPV) of the TRC against the combined results of the RT-PCR and sequencing were calculated with $95 \%$ confidence intervals $(95 \% \mathrm{CI})$.

\section{Results}

\section{Patient characteristics}

During the study period, a total of 188 patients were evaluated, comprising 92 patients in period 1 and 96 patients in period 2. Patient characteristics are shown in Table 1. Of the patients, 95 (50.5\%) visited a hospital within $24 \mathrm{~h}$ of the onset of symptoms. The percentage of patients with fatigue was significantly lower in period $1(37.0 \%)$ than in period $2(71.9 \%, P=<0.001)$. The influenza antigen test using silver amplification immunochromatography detected influenza A and B in $38(20.2 \%)$ and 39 $(20.7 \%)$ patients, respectively. The percentage of influenza A was significantly lower, while that of influenza B was significantly higher, in period 1 than in period 2 (Table 1).

\section{Comparison of TRC and RT-PCR results}

The results of the RT-PCR and TRC are shown in Table 2. In the nasal swabs, influenza A and B were detected using RTPCR in $36(19.1 \%)$ and 39 (20.7\%) patients, respectively, and were detected using TRC in $38(20.2 \%)$ and 40 (21.3\%) patients, respectively (Table 2). Of all patients, three tested negative for influenza with RT-PCR, but positive with TRC. Influenza A was detected in two of these three patients, and influenza $\mathrm{B}$ was detected in one by sequencing. Based on the combined RT-PCR and sequencing results, the Se, Sp, PPV, and NPV of the TRC were 1.000, 0.973, 0.962, and 1.000, respectively (Table 3 ).

In the gargle samples, influenza A was detected by RTPCR and TRC in 37 (38.5\%) and 35 (36.5\%) patients, respectively (Table 2). Of all patients in period 2, two tested positive for influenza A with RT-PCR, but negative with TRC. Influenza A was detected in these two patients by sequence analysis. Based on the combined RT-PCR and sequence analysis results, the Se, Sp, PPV, and NPV of the TRC in the gargle samples were 0.946, 1.000, 1.000, and 0.967 , respectively (Table 3 ). 
Table 1 Patient characteristics

\begin{tabular}{|c|c|c|c|c|c|c|c|}
\hline \multirow[t]{2}{*}{ Characteristic } & \multicolumn{2}{|c|}{ Overall $(N=188)$} & \multicolumn{2}{|c|}{ Period $1(N=92)$} & \multicolumn{2}{|c|}{ Period $2(N=96)$} & \multirow[t]{2}{*}{$P$ value } \\
\hline & $N$ & $(\%)$ & $N$ & $(\%)$ & $N$ & $(\%)$ & \\
\hline Age (average \pm S.D) & 50.5 & \pm 19.4 & 51.3 & \pm 19.5 & 49.6 & \pm 19.5 & NS \\
\hline Gender $=$ female & 103 & $(54.8 \%)$ & 58 & $(63.0 \%)$ & 45 & $(46.9 \%)$ & 0.029 \\
\hline Underlying diseases & 94 & $(50.0 \%)$ & 48 & $(52.2 \%)$ & 46 & $(47.9 \%)$ & NS \\
\hline \multicolumn{8}{|c|}{ Time since onset of symptoms } \\
\hline $0-12 \mathrm{~h}$ & 48 & $(25.5 \%)$ & 23 & $(25.0 \%)$ & 25 & $(26.0 \%)$ & NS \\
\hline $12-24 \mathrm{~h}$ & 47 & $(25.0 \%)$ & 23 & $(25.0 \%)$ & 24 & $(25.0 \%)$ & NS \\
\hline $24-48 \mathrm{~h}$ & 39 & $(20.7 \%)$ & 17 & $(18.5 \%)$ & 22 & $(22.9 \%)$ & NS \\
\hline $48-72 \mathrm{~h}$ & 19 & $(10.1 \%)$ & 7 & $(7.6 \%)$ & 12 & $(12.5 \%)$ & NS \\
\hline $72+h$ & 26 & $(13.8 \%)$ & 14 & $(15.2 \%)$ & 12 & $(12.5 \%)$ & NS \\
\hline Unknown & 9 & $(4.8 \%)$ & 8 & $(8.7 \%)$ & 1 & $(1.0 \%)$ & 0.017 \\
\hline \multicolumn{8}{|l|}{ Symptoms } \\
\hline Fever & 143 & $(76.1 \%)$ & 66 & $(71.7 \%)$ & 77 & $(80.2 \%)$ & NS \\
\hline Cough & 124 & $(66.0 \%)$ & 60 & $(65.2 \%)$ & 64 & $(66.7 \%)$ & NS \\
\hline Fatigue & 103 & $(54.8 \%)$ & 34 & $(37.0 \%)$ & 69 & $(71.9 \%)$ & $<0.001$ \\
\hline Sore throat & 100 & $(53.2 \%)$ & 46 & $(50.0 \%)$ & 54 & $(56.3 \%)$ & NS \\
\hline Nasal discharge & 99 & $(52.7 \%)$ & 47 & $(51.1 \%)$ & 52 & $(54.2 \%)$ & NS \\
\hline Headache & 70 & $(37.2 \%)$ & 35 & $(38.0 \%)$ & 35 & $(36.5 \%)$ & NS \\
\hline Arthralgia & 56 & $(29.8 \%)$ & 23 & $(25.0 \%)$ & 33 & $(34.4 \%)$ & NS \\
\hline Myalgia & 56 & $(29.8 \%)$ & 22 & $(23.9 \%)$ & 34 & $(35.4 \%)$ & NS \\
\hline Diarrhea & 11 & $(5.9 \%)$ & 3 & $(3.3 \%)$ & 8 & $(8.3 \%)$ & NS \\
\hline Nausea & 9 & $(4.8 \%)$ & 3 & $(3.3 \%)$ & 6 & $(6.3 \%)$ & NS \\
\hline \multicolumn{8}{|c|}{ Results of influenza antigen test using silver amplification immunochromatography } \\
\hline Influenza A & 38 & $(20.2 \%)$ & 3 & $(3.3 \%)$ & 35 & $(36.5 \%)$ & $<0.001$ \\
\hline Influenza B & 39 & $(20.7 \%)$ & 38 & $(41.3 \%)$ & 1 & $(1.0 \%)$ & $<0.001$ \\
\hline Negative & 111 & $(59.0 \%)$ & 50 & $(54.3 \%)$ & 60 & $(62.5 \%)$ & NS \\
\hline
\end{tabular}

S.D. standard deviation, $N S$ not significant

\section{Comparison of results between nasopharyngeal swabs and gargle samples}

In the RT-PCR testing, five patients tested negative for influenza $A$ in their nasopharyngeal swabs, but tested positive for influenza A in their gargle samples (Fig. 1). The Se, Sp, PPV, and NPV of the RT-PCR in the gargle samples were 1.000, $0.922,0865$, and 1.000, respectively. The Se, Sp, PPV, and NPV of the TRC in the gargle samples were 0.912, 0.935, 0.886 , and 0.951 , respectively.

\section{Discussion}

The novel rapid TRC assay showed great sensitivity and specificity in nasopharyngeal swabbing in both periods 1 and 2 . Based on the results of the antigen test, period 1 was considered to be the influenza B epidemic season and period 2 was considered the influenza A epidemic season (Table 1). The sensitivity and specificity of the rapid TRC assay were 1.000 and 1.000, respectively, based on the combined RT-PCR and sequencing results for both periods. There are several rapid RT-PCR assays for detection of influenza, such as the ID Now influenza A and B 2 assay (ID Now), Cobas influenza A/B nucleic acid test (Liat), and Xpert Xpress Flu assay (Xpert). The previous studies reported that the sensitivity and specificity of these methods for detecting influenza $\mathrm{A} / \mathrm{B}$ were 0.932 to $1.000 / 0.917$ to 1000 and 0.977 to $1.000 / 0.976$ to 0.998 , respectively [12-14]. These results indicate that the performance of the rapid TRC assay is comparable to that of rapid RT-PCR assays.

In the present study, the rapid TRC assay also showed great sensitivity and specificity for gargle sampling. The rapid TRC assay and RT-PCR detected influenza in more patients from gargle samples than from nasopharyngeal swabs. The sensitivity and specificity of the Rapid TRC assay were 0.946 and 1.000 , respectively. The previous studies reported that the sensitivity of Xpert and Liat for gargle sampling was 0.917 and 1.000 , respectively, in comparison with in-house RT-PCR $[15,16]$. Although there are no data on the 
Table 2 Results of RT-PCR and TRC

Table 3 Performance of TRC assay for detection of influenza

$\begin{array}{llll}\text { Item } & \frac{\text { Overall }(N=188)}{N} & \frac{\text { Period } 1(N=92)}{N} & \frac{P \operatorname{Period} 2(N=96)}{N}\end{array} \quad P$ value

RT-PCR in nasopharyngeal swab

$\begin{array}{llllllll}\text { Influenza A } & 36 & (19.1 \%) & 4 & (4.3 \%) & 32 & (33.3 \%) & <0.001 \\ \text { Subtype H1 } & 9 & (4.8 \%) & 0 & (0.0 \%) & 9 & (9.4 \%) & 0.003 \\ \text { Subtype H3 } & 27 & (14.4 \%) & 4 & (4.3 \%) & 23 & (24.0 \%) & 0.001 \\ \text { Influenza B } & 39 & (20.7 \%) & 39 & (42.4 \%) & 0 & (0.0 \%) & <0.001 \\ \text { Negative } & 113 & (60.1 \%) & 49 & (53.3 \%) & 64 & (66.7 \%) & \text { NS }\end{array}$

TRC in nasopharyngeal swab

\begin{tabular}{|c|c|c|c|c|c|c|c|}
\hline Influenza A & 38 & $(20.2 \%)$ & 4 & $(4.3 \%)$ & 34 & $(35.4 \%)$ & $<0.001$ \\
\hline Influenza B & 40 & $(21.3 \%)$ & 40 & $(43.5 \%)$ & 0 & $(0.0 \%)$ & $<0.001$ \\
\hline Negative & 110 & $(58.5 \%)$ & 48 & $(52.2 \%)$ & 62 & $(64.6 \%)$ & NS \\
\hline \multicolumn{8}{|c|}{ RT-PCR in gargle sample } \\
\hline Influenza A & N/A & & NCW & & 37 & $(38.5 \%)$ & N/A \\
\hline Subtype H1 & $\mathrm{N} / \mathrm{A}$ & & $\mathrm{NCW}$ & & 11 & $(11.5 \%)$ & N/A \\
\hline Subtype H3 & $\mathrm{N} / \mathrm{A}$ & & NCW & & 26 & $(27.1 \%)$ & $\mathrm{N} / \mathrm{A}$ \\
\hline Influenza B & N/A & & $\mathrm{NCW}$ & & 0 & $(0.0 \%)$ & N/A \\
\hline Negative & $\mathrm{N} / \mathrm{A}$ & & NCW & & 59 & $(61.5 \%)$ & $\mathrm{N} / \mathrm{A}$ \\
\hline \multicolumn{8}{|c|}{ TRC in gargle sample } \\
\hline Influenza A & $\mathrm{N} / \mathrm{A}$ & & NCW & & 35 & $(36.5 \%)$ & N/A \\
\hline Influenza B & N/A & & NCW & & 0 & $(0.0 \%)$ & $\mathrm{N} / \mathrm{A}$ \\
\hline Negative & N/A & & NCW & & 61 & $(63.5 \%)$ & N/A \\
\hline
\end{tabular}

NS not significant, N/A not applicable, $N C W$ not complied with

\begin{tabular}{llll}
\hline & Overall & Period 1 & Period 2 \\
\hline Nasopharyngeal swab & & & \\
TP & 78 & 44 & 34 \\
TN & 110 & 48 & 62 \\
FP & 0 & 0 & 0 \\
FN & 0 & 0 & 0 \\
Se $(95 \%$ CI $)$ & $1.000(0.931-1.000)$ & $1.000(0.882-1.000)$ & $1.000(0.851-1.000)$ \\
Sp $(95 \%$ CI $)$ & $1.000(0.951-1.000)$ & $1.000(0.891-1.000)$ & $1.000(0.915-1.000)$ \\
PPV (95\% CI) & $1.000(0.931-1.000)$ & $1.000(0.882-1.000)$ & $1.000(0.851-1.000)$ \\
NPV (95\% CI) & $1.000(0.951-1.000)$ & $1.000(0.891-1.000)$ & $1.000(0.915-1.000)$ \\
Gargle samples & & & \\
TP & 35 & N/A & 35 \\
TN & N/A & 59 \\
FP & N/A & 0 \\
FN & 0 & N/A & 2 \\
Se $(95 \%$ CI) & 2 & N/A & $0.946(0.818-0.993)$ \\
Sp (95\% CI) & $1.000(0.911-1.000)$ & N/A & $1.000(0.911-1.000)$ \\
PPV (95\% CI) & $1.000(0.855-1.000)$ & N/A & $1.000(0.855-1.000)$ \\
NPV (95\% CI) & $0.967(0.887-0.996)$ & N/A & $0.967(0.887-0.996)$ \\
\hline
\end{tabular}

Period 1 between January 1 and March 31, 2018; period 2 between January 1 and March 31, 2019; TP true positive; $T N$ true negative; $F P$ false positive; $F N$ false negative; $C I$ confidence interval; N/A not applicable 


\section{RT-PCR}

\begin{tabular}{cccc}
\hline & \multicolumn{3}{c}{ Nasopharyngeal swab } \\
& & Pos & Neg \\
\hline \multirow{2}{*}{ Gargle } & Pos & 32 & 5 \\
& Neg & 0 & 59 \\
\hline
\end{tabular}

TRC

\begin{tabular}{cccc}
\hline & \multicolumn{3}{c}{ Nasopharyngeal swab } \\
& & Pos & Neg \\
\hline \multirow{2}{*}{ Gargle } & Pos & 31 & 4 \\
& Neg & 3 & 58 \\
\hline
\end{tabular}

\section{Se, Sp, PPV, and NPV in gargle samples}

$\begin{array}{ccccc}\text { Se } & 1.000 & (0.842-1.000) & 0.912 & (0.763-0.981) \\ \text { Sp } & 0.922 & (0.827-0.974) & 0.935 & (0.843-0.982) \\ \text { PPV } & 0.865 & (0.712-0.955) & 0.886 & (0.733-0.968) \\ \text { NPV } & 1.000 & (0.911-1.000) & 0.951 & (0.863-0.990)\end{array}$

Fig. 1 Comparison of gargle sample and nasopharyngeal swab results. The results obtained from gargle samples and nasopharyngeal swabs during period 2 were compared. Period 2 was between January 1 and March 31, 2019

specificity of rapid RT-PCR assays, the rapid TRC assay seems to be comparable with rapid RT-PCR assays for gargle samples. In the diagnosis of influenza, nasopharyngeal swabbing is the major sampling type, but these samples are difficult to obtain and the procedure is uncomfortable for patients [17]. In addition, due to the novel coronavirus (SARS-CoV-2) pandemic, healthcare workers must wear personal protective equipment when they obtain nasopharyngeal swabs. Therefore, it is vital to develop a diagnostic method, such as the rapid TRC assay, using gargle samples, that is easy to perform, non-invasive, material saving, and safe for healthcare workers [18].

There are some limitations in this study. First, the samples were obtained from one community hospital in Nagasaki, which might have limited the generalizability of the findings. Second, we used an equipment of the novel TRC assay under development. Accordingly, we are conducting a multicenter study for the rapid TRC assay using production version. Third, the rapid TRC assay was not compared with other rapid RT-PCR assays. Since these assays have not yet been approved by the Japanese Pharmaceuticals and Medical Devices Agency, we will conduct a comparative study in the future.

In conclusion, the novel rapid TRC assay showed comparable performance to RT-PCR in the detection of influenza viruses. In addition, because it could detect influenza viruses using gargle samples, the rapid TRC assay could contribute to the diagnosis of influenza during the SARS-CoV-2 pandemic.

Author contributions All authors meet the ICMJE authorship criteria.

Funding This study was funded by Tosoh corporation.
Availability of data and materials Raw data were generated at Nagasaki University Hospital. Derived data supporting the findings of this study are available from the corresponding author on request.

\section{Declarations}

Ethical approval This study was approved by the ethics committee of Nagasaki University Hospital (approval numbers: 17121822 and 18111919)

Consent to participate Written informed consent for participation in and publication of this study was obtained from all participants before sample collection.

Consent for publication Written informed consent for publication of this study was obtained from all participants before sample collection.

Competing interests The authors declare that they have no conflict of interest.

Disclaimer The sponsor had no control over the interpretation, writing, or publication of this work.

\section{References}

1. Ishiguro T, Saitoh J, Horie R, Hayashi T, Ishizuka T, Tsuchiya S et al (2003) Intercalation activating fluorescence DNA probe and its application to homogeneous quantification of a target sequence by isothermal sequence amplification in a closed vessel. Anal Biochem 314:77-86. https://doi.org/10.1016/S0003-2697(02)00618-8 [Internet]. [cited 2020 1]; https:/www.sciencedirect.com/science/ article/pii/S0003269702006188?via\%3Dihub

2. Ishiguro T, Saitoh J, Yawata H, Otsuka M, Inoue T, Sugiura Y (1996) Fluorescence detection of specific sequence of nucleic acids by oxazole yellow-linked oligonucleotides. Homogeneous quantitative monitoring of in vitro transcription. Nucleic Acids Res 24: 
4992-4997. https://doi.org/10.1093/nar/24.24.4992 [Internet]. [cited 2020 1]; http://www.ncbi.nlm.nih.gov/pubmed/9016671

3. Mazzarelli A, Cannas A, Venditti C, D'Arezzo S, De Giuli C, Truffa S et al (2019) Clinical evaluation of TRCReady M.TB for rapid automated detection of $\mathrm{M}$. tuberculosis complex in respiratory samples. Int J Tuberc Lung Dis 23:260-264. https://doi.org/10. 5588/ijtld.18.0355 [Internet]. [cited 2020 1]; https://pubmed.ncbi. nlm.nih.gov/30808461/?from_single_result=Clinical+evaluation+ of+TRC+Ready+for+rapid+automated+detection+of+complex+ in+respiratory+samples\&expanded_search_query=Clinical + evaluation+of+TRC+Ready+for+rapid+automated+detection+of+ complex+i

4. Makino Y, Miki D, Noguchi Y, Sato H (2016) Primer for amplifying influenza virus RNA, and influenza virus detection method using the same. [Internet]. [cited 2020 1]; https://worldwide. espacenet.com/patent/search/family/056425709/publication/ JP2016131498A?q=JP6565193B

5. Hatashita R, Makino Y, Miki D, Noguchi A, Sato H Reagents for extraction and amplification of nucleic acid from virus. [Internet]. [cited 2020 15]; https://www.j-platpat.inpit.go.jp/c1800/PU/JP2017-195871/D3BADD8449A11E61DF2F46A85FCA8CF8 E5102F972C4E673D83731053DB2C6584/11/en

6. World Health Organization Global Epidemiological Surveillance Standards for Influenza [Internet]. [cited 2020 10]. https://www. who.int/influenza/resources/documents/WHO_Epidemiological_ Influenza_Surveillance_Standards_2014.pdf?ua=1

7. Mitamura K, Shimizu H, Yamazaki M, Ichikawa M, Nagai K, Katada J et al (2013) Clinical evaluation of highly sensitive silver amplification immunochromatography systems for rapid diagnosis of influenza. J Virol Methods 194:123-128. https://doi.org/10. 1016/j.jviromet.2013.08.018 [Internet]. [cited 2019 2]; https:// linkinghub.elsevier.com/retrieve/pii/S0166093413003509

8. National Institute of Infectious Diseases (2014) Manual for virus detection-influenza, 3rd edn. [Internet]. [cited 2020 15]; https:// www.niid.go.jp/niid/images/lab-manual/Influenza2014.pdf

9. Furuse Y, Tamaki R, Okamoto M, Saito-Obata M, Suzuki A, Saito $\mathrm{M}$ et al (2019) Association between preceding viral respiratory infection and subsequent respiratory illnesses among children: a prospective cohort study in the Philippines. J Infect Dis 219:197205. https://doi.org/10.1093/infdis/jiy515 [Internet]. [cited 2020 22]; http:/www.ncbi.nlm.nih.gov/pubmed/30189092

10. Kyaw Win SM, Saito R, Win NC, Lasham DJ, Kyaw Y, Lin N et al (2020) Epidemic of influenza A(H1N1)pdm09 analyzed by full genome sequences and the first case of oseltamivir-resistant strain in Myanmar 2017. PLoS One 15:e0229601. https://doi.org/10. 1371/journal.pone.0229601 [Internet]. [cited 2020 15]; https://dx. plos.org/10.1371/journal.pone.0229601

11. Kanda Y (2013) Investigation of the freely available easy-to-use software 'EZR' for medical statistics. Bone Marrow Transplant 48:452-458. https://doi.org/10.1038/bmt.2012.244 [Internet]. [cited 2020 16]; http://www.ncbi.nlm.nih.gov/pubmed/23208313
12. Kanwar N, Michael J, Doran K, Montgomery E, Selvarangan R (2020) Comparison of the ID now Influenza A \& B 2, Cobas Influenza A/B, and Xpert Xpress flu point-of-care nucleic acid amplification tests for Influenza A/B virus detection in children. J Clin Microbiol 58. https://doi.org/10.1128/JCM.01611-19 [Internet]. [cited 2020 5]; http://www.ncbi.nlm.nih.gov/pubmed/31941689

13. Mitamura K, Shimizu H, Yamazaki M, Ichikawa M, Abe T, Yasumi Y et al (2020) Clinical evaluation of ID NOW influenza A \& B 2, a rapid influenza virus detection kit using isothermal nucleic acid amplification technology - a comparison with currently available tests. J Infect Chemother 26:216-221. https://doi.org/ 10.1016/j.jiac.2019.08.015 [Internet]. [cited 2020 5]; https:// pubmed.ncbi.nlm.nih.gov/31558351/?from term $=+$ ID+Now + influenza $+A+\% 26+B+2+\&$ from_sort=date\&from pos $=3$

14. Verbakel JY, Matheeussen V, Loens K, Kuijstermans M, Goossens $\mathrm{H}$, Ieven $\mathrm{M}$ et al (2020) Performance and ease of use of a molecular point-of-care test for influenza A/B and RSV in patients presenting to primary care. Eur J Clin Microbiol Infect Dis. https://doi.org/10. 1007/s10096-020-03860-5 [Internet]. [cited 2020 5]; http://www. ncbi.nlm.nih.gov/pubmed/32172369

15. Bennett S, MacLean A, Gunson R (2019) Verification of Cepheid Xpert Xpress Flu/RSV assay for use with gargle samples, sputa and endotracheal secretions. J Hosp Infect 101:114-115. https://doi.org/ 10.1016/J.JHIN.2018.07.016 [Internet]. [cited 2020 5]; https:// www.sciencedirect.com/science/article/pii/S0195670118303803? via\%3Dihub

16. Goldstein EJ, Gunson RN (2019) In-house validation of the cobas Liat influenza A/B and RSV assay for use with gargles, sputa and endotracheal secretions. J Hosp Infect 101:289-291. https://doi.org/ 10.1016/j.jhin.2018.10.025 [Internet]. [cited 2020 5]; http://www. ncbi.nlm.nih.gov/pubmed/30408505

17. Frazee BW, Rodríguez-Hoces de la Guardia A, Alter H, Chen CG, Fuentes EL, Holzer AK et al (2018) Accuracy and discomfort of different types of intranasal specimen collection methods for molecular influenza testing in emergency department patients. Ann Emerg Med 71:509-517.e1. https://doi.org/10.1016/j. annemergmed.2017.09.010 [Internet]. [cited 2020 5]; https:// pubmed.ncbi.nlm.nih.gov/29174837/?from_single_result= Accuracy+and+Discomfort+of+Different+Types+of+Intranasal+ Specimen+Collection+Methods+for+Molecular+Influenza+ Testing+in+Emergency+Department+Patients

18. Malecki M, Lüsebrink J, Teves S, Wendel AF (2020) Pharynx gargle samples are suitable for SARS-CoV-2 diagnostic use and save personal protective equipment and swabs. Infect Control Hosp Epidemiol:1-2. https://doi.org/10.1017/ice.2020.229 [Internet]. [cited 2020 5]; http://www.ncbi.nlm.nih.gov/pubmed/ 32389132

Publisher's note Springer Nature remains neutral with regard to jurisdictional claims in published maps and institutional affiliations. 